\title{
DNA Ploidy Analyses in 218 C onsecutive Pakistani Breast C ancer Patients: Does it Add Anything?
}

\author{
Enver KHAN,${ }^{1}$ Zohair MAPARA, ${ }^{1}$ Shaista KHAN ${ }^{2}$ Naveeda ARSHAD, ${ }^{1}$ Tariq SIDDIQUI, ${ }^{3}$ Shahid PE RVEZ ${ }^{1}$ \\ ${ }^{1}$ Department of Pathology, ${ }^{2}$ D epartment of Surgery, ${ }^{3}$ Department of M edicine, The A ga K han U niversity M edical \\ Centre, $\mathrm{K}$ arachi, Pakistan
}

\begin{abstract}
An analysis was made to evaluate the significance of DNA ploidy in the biology and prognosis of breast carcinoma. This was done by estimating the correlation of DNA ploidy with other established prognostic markers of breast cancer, namely tumor size, tumor grade, lymph node metastasis and S-phase fraction. From 1995 up to year 2000 ploidy analysis was performed on 218 consecutive cases of infiltrating breast carcinoma by flow cytometry using formalin fixed paraffin embedded material. From the laboratory record, data regarding other pathological variables was retrieved. No correlation could be found between DNA ploidy and tumor grade, nor could
\end{abstract} Keywords: DNA ploidy, breast carcinoma, Pakistan there be found a correlation with tumor size. For lymph node metastasis there was a significant difference between the proportion of aneuploids and diploids having metastasis in more than 4 lymph nodes. How ever, no significant difference was found in axillary lymph node positive and negative groups when number of positive lymph nodes was not taken into account. The mean value of S-phase fraction for the aneuploids and the diploids was also insignificantly different. In conclusion D NA ploidy alone did not add much to predict tumor behaviour in terms of known pathologic variables. (Pathology Oncology Research Vol 7, No 2, 125-128, 2001)

\section{Introduction}

There is very little authentic documentation available regarding the statistics of breast cancer in Pakistan. True statistics are available for only one district of $K$ arachi, that is District South, where a Cancer R egistry has been established. A mong the few medical centers that have records regarding their pattern of malignancies, the most common type of malignancy affecting females of $\mathrm{K}$ arachi is breast cancer. In two major hospitals in Karachi $22.95 \%$ and, $20.8 \%$ of the female malignancies were that of breast cancer. ${ }^{1}$ The incidence of breast cancer in $\mathrm{K}$ arachi is estimated to be higher than any other $A$ sian population other than the J ews of Israel. ${ }^{2}$

Received: Dec 6, 2000; revised: M ay 10, 2001; accepted: M ay 28, 2001

Correspondence: Dr. Shahid PERVEZ, A ssociate Professor, Department of Pathology, The Aga Khan University Medical Centre, P.O. B ox 3500, Stadium Road, K arachi, Pakistan; Tel: 9221-4930051/1554; fax: 92-21-4934294;

E-mail: shahid.pervez@aku.edu
A few classical morphological prognostic markers such as lymph node metastasis, tumor size and tumor grade have stood the test of time and are the key prognostic markers of many tumors including breast cancer. In the last 20 years, however, many novel prognostic markers have evolved to aid in the prognosis and management of the disease. These include DNA ploidy, S-phase fraction (SPF), oncogenes, and tumor suppressor gene products. These cellular and molecular indicators are believed to not only aid in the prognosis but also suggest a host of new clinical interventions which could lead to better clinical outcome. Flow cytometry is a technique which was developed in the 80 's to analyze tissues on a cell to cell basis. It is now possible to determine whether the DNA of each cell is normal (diploid versus nondiploid) and the fraction of cells actively synthesizing DNA. The degree of departure from normal DNA content is calculated as the DNA index (DI). By definition, a diploid tumor has a DI of 1.0. (Standard deviation, SD 0.9-1.10) Inspite of numerous studies suggesting usefulness of ploidy and SPF estimation of neoplastic cells, ploidy and proliferative activity is yet to prove to have a substantial role in the diagnosis and prognosis of 
most tumors on regular basis. In breast cancer since the very beginning, conflicting data has surfaced regarding its correlation with other established prognostic parameters. It is now becoming obvious that DNA ploidy can only possibly come up as a prognostic marker if detection is carried out in stage I and stage II. It will obviously have very little significance in stage III and stage IV disease.

Initially DNA ploidy was carried out with much enthusiasm by many pathologists, however, its significance in the present day pathology and oncology practice is in question. Its role in predicting survival has also become controversial. Some studies have found significant difference in survival between patients with aneuploidy and those with diploidy, ${ }^{4}$ with diploids having a better prognosis. However there are other studies which contradict these findings. 5

In addition in later studies racial differences were also highlighted. A study carried out by Shiao et $\mathrm{al}^{7}$ in U nited States reached the conclusion that DNA ploidy holds dissimilar significance as a prognostic marker amongst Blacks and Whites. A study carried out by Wong et $\mathrm{al}^{6}$ in A ustralia and another carried out by $C$ hen et $\mathrm{al}^{8}$ in Taiwan revealed different and even conflicting results. A nother study ${ }^{9}$ compares whites, blacks and A sians living in the same locality. It follows that the significance of DNA ploidy as a prognostic variable also depends on the race of the population under study, and hence this study was carried out to evaluate the significance of this marker in our setting where the prevalence and particularly the age group is much different from Western data (median age at the time of diagnosis is approximately 49 years).

In this study, breast tumors which were brought to the A ga $\mathrm{K}$ han university pathology laboratory, K arachi, Pakistan, were analyzed and DNA ploidy was compared with the other established morphological parameters which include tumor size, histological grade and axillary lymph node metastasis.

\section{Materials and M ethods}

\section{Selection of cases}

In this study 218 consecutive cases of breast cancer were examined from formalin fixed paraffin embedded tissue blocks. Only those blocks were selected which on screening showed a good proportion of representative tissue. From the laboratory record, data regarding other pathological variables was retrieved.

\section{Sample Preparation}

Three to five $25 \mu \mathrm{m}$ thick sections were cut from routinely fixed, paraffin embedded tissue blocks for each case. Sections were dewaxed in two changes (2x10 minutes) of xylene and rehydrated in $100,90,70$ and $50 \%$ al cohol for 10 minutes each. The sections were then rinsed in PBS x 10 minutes and incubated in $0.5 \%$ pepsin solution at $\mathrm{pH} 1.5$ at $37^{\circ} \mathrm{C}$ for 30 minutes. Hypodermic needles of 40 and 25 bore were then used to break up the tissue. Released nuclei were then spun, washed and cytopreps made to check the condition of nuclei. Nuclei were then stained with propidium iodide in isoton $(250 \mu \mathrm{g} / \mathrm{ml})$ containing $1 \mathrm{mg} / \mathrm{ml} \mathrm{RNA}$ ase for 30 minutes at $4{ }^{\circ} \mathrm{C}$ before analyzing on FACScan (B ecton-Dickinson)

\section{F low Cytometry}

Samples were analyzed on FACScan flow cytometer using the software MODFIT, Flow cytometric data was acquired and displayed in standard two parameter dot plots using FL 2 width and FL 2 area as the axes. This allowed to draw gates in which debris below the first Go/G 1 distribution and particles with extended time in flight (presumed doublets) were excluded from analysis using carefully defined and standardized gating criteria. FL 2 area signals were then used to generate single parameter DNA histograms. Specimens were rejected if the median half peak coefficient of variation (CV) of the diploid peak was more than 5. Total of 10,000 nuclei were counted in each case.

\section{Results}

The population under study were females which were known cases of breast cancer predominantly infiltrating ductal carcinoma (IDC) of breast. The sample size was 218. Out of $218,53(24 \%)$ tumors showed aneuploidy and 165 (76\%) were diploid. The DI was described as either diploid, (DI less than 1.10) or else aneuploid.The distribution of DI of the 218 breast cancer specimens is given in Figure 1. A mongst the aneuploids, 12 cases were further classified as near diploid (DI between 1.10 and 1.30) and 7 as tetraploid (DI between 1.90 and 2.10) cases. The mean DI for the aneuploids was 1.632 with a median value of 1.515 . In the aneuploids $62 \%$ of the cases had axiallary

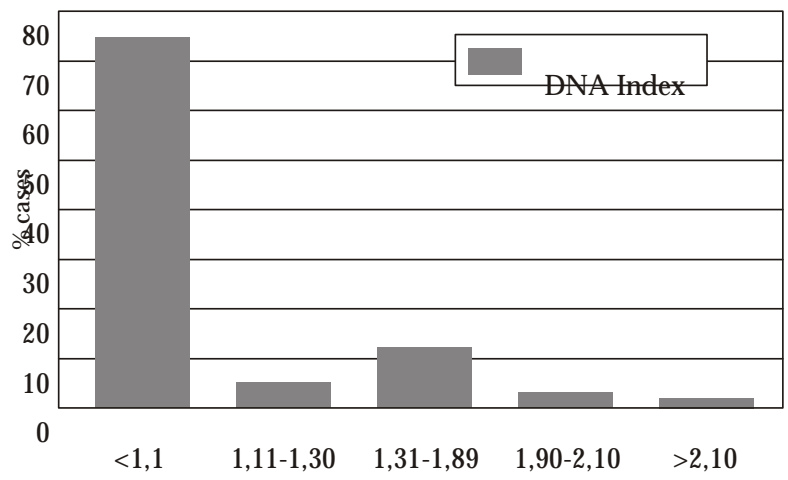

Figure 1. Distribution of DNA indices of 218 breast cancer specimens 


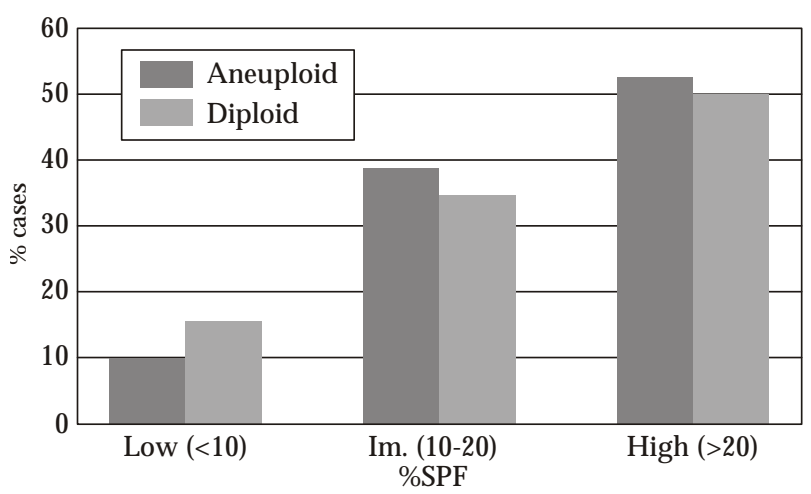

Figure 2. Ploidy distribution of breast cancer in relation to S-phase fraction

lymph node metastasis compared to $54 \%$ of the diploids which showed lymph node positivity ( $p$-value $>0.05$ ).

There was data available regarding the SPF for 178 cases from our study group. Out of these 178 cases, there were 21 cases of aneuploidy and 157 cases of diploidy. The overall mean value obtained for the SPF was $21.51 \%$. For the aneuploids the mean SPF was $24.66 \%$ with a median value of $26.48 \%$.

\section{Table 1. Relationship between ploidy status and size}

\begin{tabular}{lccc}
\hline Size & A neuploid N 0.\% & Diploid N 0.\% & p-value \\
\hline 1 & $1(2.1)$ & $4(2.7)$ & 0.75 \\
2 & $35(72.9)$ & $109(71.7)$ & 0.8711 \\
3 & $12(25.0)$ & $39(25.6)$ & 0.9274 \\
\hline
\end{tabular}

$\mathrm{N}$; A neuploids $=48$; Diploids $=152$

Code for size: (1) $0-1 \mathrm{~cm}$; (2) $>1$ to $5 \mathrm{~cm}$; (3) $>5 \mathrm{~cm}$

Table 2. Relationship between ploidy status and grade

\begin{tabular}{lccc}
\hline Garde & A neuploid N 0.\% & Diploid N 0.\% & p-value \\
\hline I & $5(11.9)$ & $11(7.6)$ & 0.579 \\
II & $28(66.67)$ & $93(64.6)$ & 0.803 \\
III & $9(21.46)$ & $40(27.8)$ & 0.411 \\
\hline
\end{tabular}

$\mathrm{N}$; A neuploids $=42$; Diploids $=144$

Table 3. Relationship between ploidy status and lymph node metastasis

\begin{tabular}{lccl}
\hline $\begin{array}{l}\text { Lymphnode } \\
\text { metastasis }\end{array}$ & A neuploid N 0 \% & Diploid N 0. \% & p-value \\
\hline 0 & $18(38.3)$ & $68(46)$ & 0.3575 \\
1 & $10(21.7)$ & $42(28.4)$ & 0.337 \\
2 & $19(40.4)$ & $38(25.6)$ & 0.053 \\
\hline
\end{tabular}

$\mathrm{N}$; A neuploids $=47$; Diploids $=148$

Code: (0) No of metastasis; (1) metastasis in 1-3 lymph nodes; (2) metastasis in 4 or more lymph nodes
For the diploids the mean value of SPF was $21.09 \%$ with a median value of $19.6 \%$. A mong all the 178 cases, the SPF was below $10 \%$ (low) in only $14.04 \%$ of the cases, between $10 \%$ and $20 \%$ (intermediate) in $35.4 \%$ of the cases, and more than $20 \%$ (high) in $50.56 \%$ of the cases. When the mean values of the SPF for the aneuploids and the diploids were compared, the difference was found to be statistically insignificant with a p-value of 0.689 . The graph in Figure 2. shows the results obtained when proportions of aneuploids and diploids were compared in each of the three categories of SPF, namely low $(<10 \%)$, intermediate ( $10 \%$ to $20 \%$ ) and high (>20\%).

Univariate analysis of DNA ploidy was done in relation to tumor size, histological grade and lymph node metastasis.

Size of the tumor was divided into three categories, and coded as $1(0-1 \mathrm{~cm}), 2(>1-5 \mathrm{~cm})$ and $3(>5 \mathrm{~cm})$. The proportions of aneuploids falling in each category were compared with proportions of diploids falling in the same categories respectively. For all three groups, the $p$-values were insignificant. (Table 1.)

The proportion of aneuploids and diploids for each grade were also compared and for all the three grades, (grade I, II and III), there was no significant difference. (Table 2.)

Regarding lymph node status, the division was no metastasis (0), metastasis with 1-3 lymph nodes (1) and metastasis with 4 or more lymph nodes (2). On comparing the proportions of aneuploids and diploids falling in each categories, no correlation was seen in the first two categories, (with no metastasis and with metastasis in 1-3 lymph nodes). However in the third category (met. in 4 or more lymph nodes), a weak correlation was seen with a pvalue equal to 0.05 . (Table 3.)

\section{Discussion}

The aim of our study was to estimate the significance of DNA ploidy as a prognostic marker in our setting of breast cancer patients. The study group is representative of the population of Pakistan, not just because the samples were collected from $\mathrm{K}$ arachi, a cosmopolitan city with people from all races present in the country, but al so because the laboratory receives samples from all over Pakistan.

Our results show that the aneuploids were $24 \%$, whereas the diploids were $76 \%$. This ratio is different from some studies carried out in other parts of the world, ${ }^{10,15}$ which showed a majority of aneuploids amongst their sample population. This suggests that there might be a role of inter-racial diversity.

The SPF compared with DNA ploidy revealed that there was not a very significant difference between the mean SPF of the aneuploids and the diploids. However, the common trend of more aneuploids with high SPF and more diploids with low SPF was noted in our study as well. Nonetheless this difference is very small. Since SPF is a more established prognostic marker of breast cancer, the absence of a signifi- 
cant difference in the SPF of aneuploids and diploids leads to the conclusion that both the conditions of DNA ploidy are not indicative of prognosis as such. M any other studies however have found significant differences. ${ }^{5,11-16}$ In our population the mean SPFobserved was quite high, $(21.51 \%)$ a feature al so noted in a similar study carried out in India. ${ }^{15}$

For size, no significant correlation could be determined in our study. This is in Contradiction to a few studies, ${ }^{11,17-20}$ however many studies have reached the same conclusion as ours as well. ${ }^{15,21}$ For grade no correlation could be seen with DNA ploidy, similar to another study, ${ }^{15}$ but we noticed a high proportion of tumors presenting characteristics of grade II carcinoma in both aneuploids and diploids. Few studies however have found a correlation between grade and DNA ploidy. 4,6,17,22

A nother factor that might be brought into consideration here is that in our setting a lot of cases are brought to the hospital only at an advanced stage, for multiple reasons, when lymph node metastasis is a more significant feature than size. In coherence to this reasoning, we did establish a correlation of DNA ploidy with lymph node metastasis where the proportion of aneuploids which had 4 or more lymph nodes positive were significantly higher than diploids, like in another study. ${ }^{11} \mathrm{M}$ any studies carried out in other settings do not agree with us. ${ }^{15,19,20,21}$

In summary over the last several years conflicting data has surfaced regarding the extent of significance of DNA ploidy with breast cancer, and there could be many possible explanations. Among these interracial differences could be very important. These differences have been noticed in earlier studies. ${ }^{6-9} \mathrm{~A}$ nother feature could be that in different laboratories, the methodology of sample staining and sample preparation for running the flow cytometry test could be different. The difference in classification could also be responsible.

We conclude by suggesting a larger study which incorporates not only other prognostic markers that have become available of late but also to include the measures of survival and disease free survival. We did not include survival in our study because flow cytometry was introduced in our laboratory only five years ago and significant survival data is not yet available of these patients. Now as other prognostic markers are also available their significance should be determined for our population so that it provides a guidance whether or not to make popular a certain particularly expensive test in our setting where besides other considerations, one of the most important consideration is certainly the cost to the patient.

\section{References}

1. ${ }^{2}$ Naila $Z$, Azhar M , Parveen A, et al: Pattern of malignant tumors in K arachi- is it different? JCPSP 10:338-341, 2000.

2. ${ }^{2 B}$ hurgri $Y$, Bhurgi A, Hassan SH, et al: Cancer Incidence in Karachi, Pakistan: first results form $\mathrm{K}$ arachi Cancer Registry. Int J Cancer 85:325-329, 2000.
3.2Brower ST, Tartter PI, Ahmed S, et al: Proliferative indices and oncoprotein expression in benign and malignant breast biopsies. A nn Surg Oncol 2:416-423, 1995.

4. U tada $Y$, Yoshimoto $M, K$ asumi $F$, et al: Relationship between DNA ploidy and survival in breast cancer. Gan To Kagaku Ryoho 25 Suppl: 431-435, 1998.

5. ${ }^{2}$ Camplejohn RS, Ash CM, Gillett CE, et al: The prognostic significance of DNA flow cytometry in breast cancer: results from 881 patients treated in a single centre. B J Cancer 71:140-145, 1995.

6. ${ }^{2}$ Wong SW, Rangan AM, Bilous AM, et al: The value of S-phase and DNA ploidy analysis as prognostic markers for node-negative breast cancer in the A ustralian setting. Pathology 31:90-94, 1999.

7.25hiao YH, Chen VW, Lehmann HP, et al: Patterns of DNA ploidy and S-phase fraction associated with breast cancer survival in blacks and whites. Clin Cancer Res. 3:587-592, 1997.

$8 .{ }^{2} \mathrm{C}$ hen CM , C hang HT, M ok KT, et al: A nalysis of prognostic factors in Chinese women with breast cancer in southern Taiwan. Chung Hua I Hsueh Tsa Chih Taipei 62:717-723, 1999.

9. K Krieger N, van den Eeden SK, Zava D, et al: Race/ethnicity, social class and prevalence of breast cancer prognostic biomarkers: A study of white, black, and A sian women in the San Francisco bay area. Ethn Dis 7:137-149, 1997.

10. ${ }^{2}$ Stanton PD, Cooke TG, 0 akes SJ, et al: Lack of prognostic significance of DNA ploidy and $S$ phase fraction in breast cancer. BrJ Cancer 66:925-929, 1992.

11.25tal 0, Brisfors A, Carstensen J, et al: Relationships of DNA ploidy, S-phase fraction and hormone receptor status to tumor stage in breast cancers detected by population screening. The South-East Sweden B reast Cancer Group. Int J Cancer 51:2833, 1992.

12. ${ }^{2}$ eichter GE, Mueller $A$, Kaufmann $M$, et al: Correlation of DNA flow cytometric results and other prognostic factors in primary breast cancers. Int J Cancer 41:823-828, 1988.

13. ${ }^{2}$ allioniemi $O P, B$ anco $G$, Alavaikko $M$, et al: Improving the prognostic value DNA flow cytometry in Breast Cancer by combining DNA index and S-Phase fraction. Cancer 62:21832190, 1988.

13. ${ }^{2}$ D ressler $L G$, Seamer $L C, O$ wens $M A$, et al: A flow cytometry and prognostic factors in 1331 frozen breast cancer specimens. Cancer 61:420-427, 1988.

14. ${ }^{2}$ Redkar AA, Balsara BR, Bhisey AN, et al: DNA analysis of breast cancer by flow cytometry and correlation with other prognostic parameters. Indian J M ed Res 96:223-229, 1992.

15. ${ }^{2} \mathrm{D}$ ittadi R, Calderazzo F, Cabrelle A, et al: C-erbB-2/neu protein expression, DNA ploidy and S phase in breast cancer. Cell Prolif 29:403-412, 1996.

16. ${ }^{2}$ Wenger $C R$, Beardslee $S$, O wens MA, et al: DNA ploidy, Sphase, and steroid receptors in more than 127,000 breast cancer patients. B reast Cancer Res Treat 28:9-20, 1993.

17.'B eerman H, K luin PM, Hermans J, et al: Prognostic Significance of DNA ploidy in a series of 690 primary breast cancer patients. Int J Cancer 45:34-945, 1990.

18.2F allenius $A G$, Franzen $S A$, Auer GU: Predictive $V$ alue of Nuclear DNA content in Breast Cancer in relation to Clinical and M orphological factors. Cancer 62:521-530, 1988.

19. ${ }^{2}$ Ewers $S B$, Langstrom E, Baldetorp B, et al: Flow cytometric DNA analysis in Primary B reast Carcinoma and Clinicopatholgical Correlation. Cytometry 5:408-419, 1984.

20.2Bergers E, van Diest PJ, Baak J P: Cell cycle analysis of 932 flow cytometric DNA histograms of fresh frozen breast carcinoma material. Correlations between flow cytometric, clinical, and pathologic variables. Cancer. 1996; 77(11): 2258-66.

21.2Brotherick I, Shenton BK, Cowan WK, et al: The relationship between flow-cytometric and immunohistochemically detected c-erbB-2 expression, grade and DNA ploidy in breast cancer. Cancer Immunol Immunother 41:137-145, 1995. 